\title{
Active Suspension in Integrated Vehicle Control
}

\author{
Péter Gáspár, Zoltán Szabó and József Bokor \\ Computer and Automation Research Institute \\ Hungary
}

\section{Introduction}

These days road vehicles contain several individual active control mechanisms that solve a large number of control tasks. These components are often highly nonlinear, which are modelled as hybrid systems. An example is the semiactive/active suspension system, which can be modelled as a nonlinear dynamics augmented with an actuator that has a bimodal dynamics, i.e. a closed loop switching system with two modes. Moreover, in traditional control systems the vehicle functions to be controlled are designed and implemented separately. Although in the design of the individual control components only a subset of the full vehicle dynamics is considered these components influence the entire vehicle. Thus in the operation of these autonomous control systems interactions and conflicts may occur that might overwrite the intentions of the designers concerning the individual performance requirements. The aim of the integrated control methodologies is to combine and supervise all controllable subsystems affecting vehicle dynamic responses in order to ensure the management of resources. The solution might be the integration of the control logic of subsystems.

Active suspensions are used to provide good handling characteristics and improve ride comfort while harmful vibrations caused by road irregularities and on-board excitation sources act upon the vehicle. The performance of suspension systems is assessed quantitatively in terms of several parameters: passenger comfort, suspension deflection, tire load variation and energy consumption, see Gillespie (1992); Sharp \& Crolla (1987). In order to improve passenger comfort it is important to keep the effects of the road disturbance on the heave acceleration small. Structural features of the vehicle place a hard limit on the amount of suspension deflection available for reducing the acceleration of the vehicle body. Hence it is also important to keep the effect of the disturbance on the suspension deflection sufficiently small. In order to reduce the dynamic tire load deflection, the effects of the disturbance on tire deflection should also be kept small. The control force limitation is incorporated into the design procedure in order to avoid large control forces.

Applying a braking force decelerates the vehicle. Additionally, the role of the active brake is to apply unilateral braking since it reduces the lateral tire forces directly Chen \& Peng (2001); Palkovics et al. (1999). This feature provides a redundancy in affecting the lateral dynamics. However, using the active brake might have unwanted side effects as the modification of the yaw dynamics and of the longitudinal direction of the vehicle. Therefore the use of the active brake is preferred only in emergencies.

In this paper a control structure that integrates active suspensions and an active brake is proposed to improve the safety of vehicles. On the global level the active suspension system is primarily designed to improve passenger comfort and road holding. However, it is able to 
generate a stabilizing moment to balance an overturning moment during vehicle maneuvers in order to reduce the rollover risk. The controlled braking system is activated only when the vehicle comes close to rolling over. During abrupt brakings pitch dynamics increases significantly. The active suspension is also able to generate a moment and improve the pitch stability of the vehicle.

Rollover prevention is a safety feature. The combination of the active suspension and active brake handles the emergency situation provided that the active suspension component is fully functional. In order to enhance safety the reconfigurable control is extended with a fault-tolerant property in order to guarantee performances even if a hydraulic actuator fault occurs in the active suspension system. The solution of the fault-tolerant operation requires the reconfigurability of the active brake.

The control design of switched systems is involved at two levels: at the suspension actuator level a tracking controller is designed for a bimodal system together with a fault detection filter.

This paper presents the application of the Linear Parameter Varying (LPV) method for the design of integrated vehicle control systems, in which several active components are used in co-operation. In the control design besides performance specifications and uncertainties, the fault information can be taken into consideration. By monitoring suitable scheduling parameters in the LPV control, the reconfiguration of the control systems can be achieved, conflict between performance demands can be avoided and faults (loss in effectiveness) can be handled. This level provides the reference signal for the low-level actuator design and it also constitutes the supervisor controller for the reconfiguration. By using the LPV method the designed controller guarantees the desired stability and performance demands of the closedloop system. The operation of the control systems is demonstrated through various simulation vehicle maneuvers.

The structure of the paper is the following: the global chassis model containing both the vertical and later dynamics is presented in Section 2. It is followed by a detailed formulation of the control problem in Section 3. The actuator dynamics is considered in Section 4. where a tracking control is designed. Possible faults of the suspension actuator are detected by using the FDI filter of Section 5. The proposed method is demonstrated through a series of simulation examples in Section 6. Finally some conclusion remarks are formulated in Section 7.

\section{Global chassis model}

The class of finite dimensional linear systems, whose state space entries depend continuously on a time varying parameter vector, $\rho(t)$, is called LPV. The trajectory of the vector-valued signal, $\rho(t)$ is assumed not to be known in advance, although its value is accessible (measured) in real time and is constrained a priori to lie in a specified bounded set. The idea behind using LPV systems is to take advantage of the casual knowledge of the dynamics of the system, see Becker \& Packard (1994); Leith \& Leithead (2000); Rough \& Shamma (2000); Wu (2001). The formal definition of an LPV system is given below:

For a compact subset $\mathcal{P} \subset \mathcal{R}^{S}$, the parameter variation set $\mathcal{F}_{\mathcal{P}}$ denotes the set of all piecewise continuous functions mapping $\mathcal{R}$ (time) into $\mathcal{P}$ with a finite number of discontinuities in any interval. The compact set $\mathcal{P} \subset \mathcal{R}^{S}$, along with continuous functions $A: \mathcal{R}^{S} \rightarrow \mathcal{R}^{n \times n}$ and $B: \mathcal{R}^{S} \rightarrow \mathcal{R}^{n \times n_{u}}$ represents an $n^{\text {th }}$ order LPV system $G(\rho)$ whose dynamics evolve as

$$
\dot{x}=A(\rho) x+B(\rho) u,
$$


where $\rho \in \mathcal{F}_{\mathcal{P}}$. One characteristics of the LPV system is that it must be linear in the pair formed by the state vector $x$, and the control input vector $u$. The matrices $A$ and $B$ are generally nonlinear functions of the scheduling vector $\rho$.

Due to the complexity of a vehicle model two models are formalized. One model is suitable for designing the suspension system and it also takes the vertical dynamics into consideration. The other model is used to design lateral dynamics and the brake system. Connection between the two models is achieved by the application of the integrated control system.

In order to describe the vertical dynamics a full-car model, which is shown in Figure 1, is used that comprises five parts: the sprung mass and four unsprung masses at the left and right hand side at the front and rear. All the suspensions are modelled as an ensemble of a spring, a damper and an actuator to generate a pushing force between the body and the axle. The suspension stiffness and the tire stiffness are denoted by $k_{s}$ and $k_{t}$ while front and rear suspension dampers are denoted by $b_{s}$, respectively. Let the front and rear displacement of the sprung mass on the left and right side be denoted by $x_{1 f l}, x_{1 r l}$ and $x_{1 f r}, x_{1 r r}$. Let the front and rear displacement of the unsprung mass on the left and right side be denoted by $x_{2 f l}, x_{2 r l}$, $x_{2 f r}$, and $x_{2 r r}$. In the full-car model, the disturbances, $w_{f l}, w_{r l}, w_{f r}, w_{r r}$ are caused by road irregularities. The input signals, $f_{f l}, f_{r l}, f_{f r}, f_{r r}$ are generated by the actuators. The system

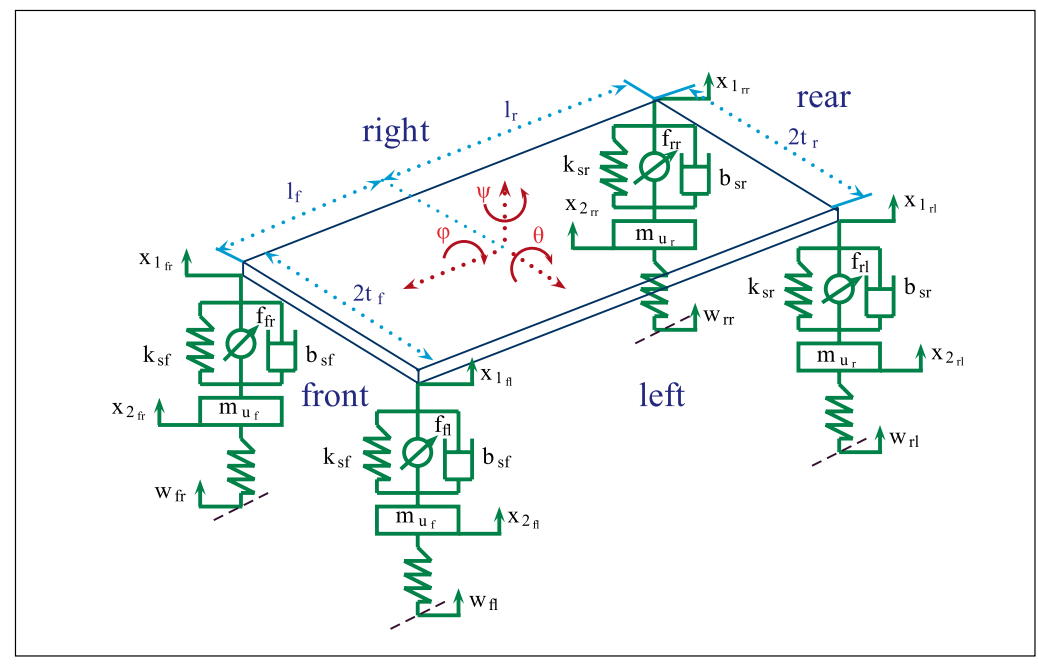

Fig. 1. Vertical model of the vehicle

equations correspond to a seven degrees-of-freedom full-car vehicle model. The sprung mass is assumed to be a rigid body and has freedoms of motion in the vertical, pitch and roll directions. The $x_{1}$ is the vertical displacement at the center of gravity, $\theta$ is the pitch angle and $\phi$ is the roll angle of the sprung mass, respectively. Each unsprung mass has freedom of motion in the vertical direction, $x_{2 f l}, x_{2 r l}, x_{2 f r}, x_{2 r r}$. The vehicle dynamical model, i.e. the heave motion, 
the pitch motion, the roll motion, the front and rear tires, is as follows:

$$
\begin{aligned}
& m_{s} \ddot{x}_{1}=F_{k f l}+F_{k f r}+F_{k r l}+F_{k r r}+F_{b f l}+F_{b f r}+F_{b r l}+F_{b r r} \\
&-f_{f l}-f_{f r}-f_{r l}-f_{r r}, \\
& I_{\theta} \ddot{\theta}=l_{f} F_{k f l}+l_{f} F_{k f r}-l_{r} F_{k r l}-l_{r} F_{k r r}+l_{f} F_{b f l}+l_{f} F_{b f r}-l_{r} F_{b r l}+l_{r} F_{b r r} \\
&-l_{f} f_{f l}-l_{f} f_{f r}+l_{r} f_{r l}+l_{r} f_{r r}, \\
& I_{\phi} \ddot{\phi}=t_{f} F_{k f l}-t_{f} F_{k f r}+t_{r} F_{k r l}-t_{r} F_{k r r}+t_{f} F_{b f l}-t_{f} F_{b f r}+t_{r} F_{b r l}-t_{r} F_{b r r} \\
&-t_{f} f_{f l}+t_{f} f_{f r}-t_{r} f_{r l}+t_{r} f_{r r}, \\
& m_{u f} \ddot{x}_{2_{f l}}=-F_{k f l}-F_{t f l}-F_{b f l}+f_{f l}, \\
& m_{u f} \ddot{x}_{2_{f r}}=-F_{k f r}-F_{t f r}-F_{b f r}+f_{f r}, \\
& m_{u r} \ddot{x}_{2_{r l}}=-F_{k r l}-F_{t r l}-F_{b r l}+f_{r l}, \\
& m_{u r} \ddot{x}_{2_{r r}}=-F_{k r r}-F_{t r r}-F_{b r r}+f_{r r},
\end{aligned}
$$

where the following linear approximations are applied:

$$
\begin{array}{cl}
x_{1_{f l}}=x_{1}+l_{f} \theta+t_{f} \phi, & x_{1_{f r}}=x_{1}+l_{f} \theta-t_{f} \phi, \\
x_{1_{r l}}=x_{1}-l_{r} \theta+t_{r} \phi, & x_{1_{r r}}=x_{1}-l_{r} \theta-t_{r} \phi .
\end{array}
$$

The suspension damping force and the suspension spring force, respectively, are as follows:

$$
\begin{aligned}
& F_{b i j}=b_{s}^{l}\left(\dot{x}_{2 i j}-\dot{x}_{1 i j}\right)-b_{s}^{s y m}\left|\dot{x}_{2 i j}-\dot{x}_{1 i j}\right|+b_{s}^{n l} \sqrt{\left|\dot{x}_{2 i j}-\dot{x}_{1 i j}\right|} \operatorname{sgn}\left(\dot{x}_{2 i j}-\dot{x}_{1 i j}\right), \\
& F_{k i j}=k_{s}^{l}\left(x_{2 i j}-x_{1 i j}\right)+k_{s}^{n l}\left(x_{2 i j}-x_{1 i j}\right)^{3},
\end{aligned}
$$

and $f_{i j}$ are the forces of the actuator, where $i j \in\{f l, f r, r l, r r\}$. Here, parts of the nonlinear suspension damper $b_{s}$ are $b_{s}^{l}, b_{s}^{n l}$ and $b_{s}^{\text {sym }}$. The $b_{s}^{l}$ coefficient affects the damping force linearly while $b_{s}^{n l}$ has a nonlinear impact on the damping characteristics. $b_{s}^{\text {sym }}$ describes the asymmetric behavior of the characteristics. Parts of the nonlinear suspension stiffness $k_{s}$ are a linear coefficient $k_{s}^{l}$ and a nonlinear one, $k_{s}^{n l}$. The tire force is approximated by a linear model:

$$
F_{t i j}=k_{t}\left(x_{2 i j}-w_{i j}\right) .
$$

The state vector $x$ is selected as follows:

$$
x_{s}=\left[\begin{array}{llll}
q & x_{u} & \dot{q} & \dot{x}_{u}
\end{array}\right]^{T}
$$

with $q=\left[\begin{array}{lll}x_{1} & \theta & \phi\end{array}\right]^{T}$ and $x_{u}=\left[\begin{array}{llll}x_{2 f l} & x_{2 f r} & x_{2 r l} & x_{2 r r}\end{array}\right]^{T}$. The state space representation of the $L P V$ model is as follows:

$$
\dot{x}_{s}=A_{s}\left(\rho_{s}\right) x_{s}+B_{1 s_{v}}\left(\rho_{s}\right) d_{s}+B_{2 s_{v}}\left(\rho_{s}\right) u_{s},
$$

where

$$
u_{s}=\left[\begin{array}{llll}
f_{f l} & f_{f r} & f_{r l} & f_{r r}
\end{array}\right]^{T} .
$$


The disturbance is $d_{s}=\left[\begin{array}{llll}w_{f l} & w_{r l} & w_{f r} & w_{r r}\end{array}\right]$. Variables concerning the front and rear displacement between the sprung mass and the unsprung mass on the left and right side and their velocities are selected as scheduling variables:

$$
\rho_{s}=\left[\begin{array}{ll}
\rho_{b i j} & \rho_{k i j}
\end{array}\right]^{T}, \quad i j \in(f l, f r, r l, r r)
$$

where

$$
\begin{aligned}
& \rho_{b i j}=\dot{x}_{2 i j}-\dot{x}_{1 i j}, \\
& \rho_{k i j}=x_{2 i j}-x_{1 i j}
\end{aligned}
$$

The scheduling variables $\rho_{b i j}$ depend on the relative velocity, while the scheduling variables $\rho_{k i j}$ depend on the relative displacement. In practice, the relative displacement is a measured signal. The relative velocity is then determined by numerical differentiation from the measured relative displacement.

Figure 2 illustrates the combined yaw-roll dynamics of the vehicle modelled by a three-body system, in which $m_{S}$ is the sprung mass, $m_{u, f}$ is the unsprung mass at the front including the front wheels and axle, and $m_{u, r}$ is the unsprung mass at the rear with the rear wheels and axle. $\beta$ denotes the side slip angle of the sprung mass, $\psi$ is the heading angle, $\phi$ is the roll angle, $\dot{\psi}$ denotes the yaw rate and $\theta$ the pitch angle. The roll angle of the unsprung mass at the front and at the rear axle are denoted by $\phi_{t, f}$ and $\phi_{t, r}$, respectively. $a_{y}$ denotes the lateral acceleration and $z_{S}$ is the heave displacement while $v$ stands for the forward velocity.

In the vehicle modelling the motion differential equations of the yaw-roll dynamics of the single unit vehicle, i.e. the lateral dynamics, the yaw moment, the roll moment of the sprung mass, the roll moment of the front and the rear unsprung masses, are formalized.

$$
\begin{aligned}
m v(\dot{\beta}+\dot{\psi})-m_{s} h \ddot{\phi} & =F_{y, f}+F_{y, r}, \\
-I_{x z} \ddot{\phi}+I_{z z} \ddot{\psi} & =F_{y, f} l_{f}-F_{y, r} l_{r}+l_{w} \Delta F_{b} \\
\left(I_{x x}+m_{s} h^{2}\right) \ddot{\phi} & -I_{x z} \ddot{\psi}=m_{s} g h \phi+m_{s} v h(\dot{\beta}+\dot{\psi}) \\
& -k_{f}\left(\phi-\phi_{t, f}\right)-b_{f}\left(\dot{\phi}-\dot{\phi}_{t, f}\right)+u_{f} \\
& -k_{r}\left(\phi-\phi_{t, r}\right)-b_{r}\left(\dot{\phi}-\dot{\phi}_{t, r}\right)+u_{r}, \\
-h_{r} F_{y, f} & =m_{u, f} v\left(h_{r}-h_{u, f}\right)(\dot{\beta}+\dot{\psi})+m_{u, f} g h_{u, f} \phi_{t, f}-k_{t, f} \phi_{t, f} \\
& +k_{f}\left(\phi-\phi_{t, f}\right)+b_{f}\left(\dot{\phi}-\dot{\phi}_{t, f}\right)+u_{f}, \\
-h_{r} F_{y, r} & =m_{u, r} v\left(h_{r}-h_{u, r}\right)(\dot{\beta}+\dot{\psi})-m_{u, r} g h_{u, r} \phi_{t, r}-k_{t, r} \phi_{t, r} \\
& +k_{r}\left(\phi-\phi_{t, r}\right)+b_{r}\left(\dot{\phi}-\dot{\phi}_{t, r}\right)+u_{r} .
\end{aligned}
$$

The detailed derivation of the equations of the yaw-roll dynamics of the single unit vehicle can be found in Sampson \& Cebon (2003).

The lateral tire forces $F_{y, i}$ in the direction of velocity at the wheel ground contact points are approximated proportionally to the tire side slip angle $\alpha_{i}$ :

$$
F_{y, f}=\mu C_{f} \alpha_{f}, \quad F_{y, r}=\mu C_{r} \alpha_{r} .
$$

The $C_{i}$ is the tire side slip constant and $\alpha_{i}$ is the tire side slip angle associated with the front and rear axles. The chassis and the wheels have identical velocities at the wheel ground contact points. The velocity equations for the front and rear wheels in the lateral and in the 


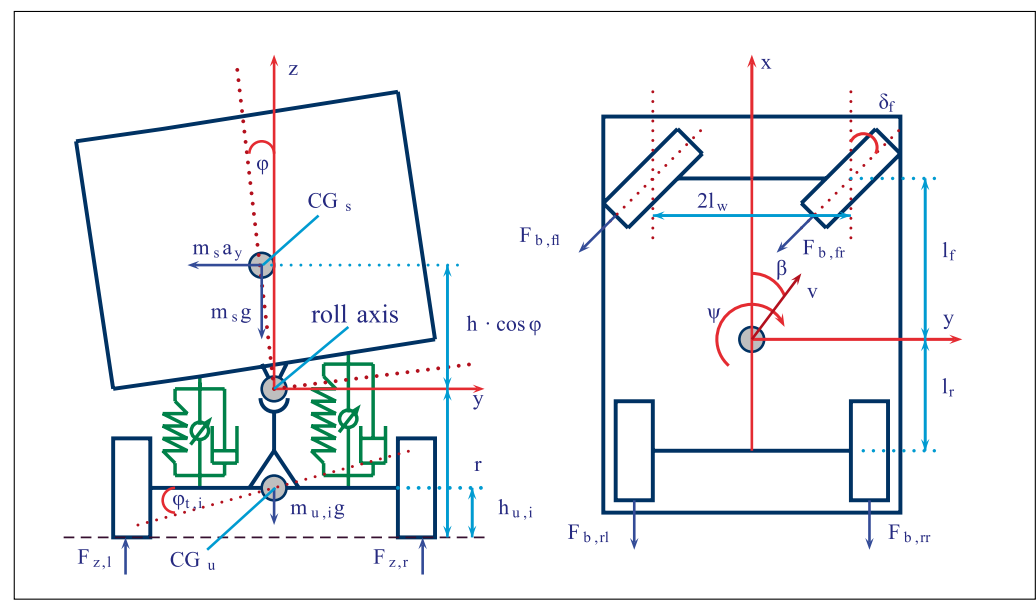

Fig. 2. Yaw and roll model of the vehicle

longitudinal directions are as follows:

$$
\begin{aligned}
& v_{w, f} \sin \left(\delta_{f}-\alpha_{f}\right)=l_{f} \cdot \dot{\psi}+v \sin \beta, \quad v_{w, f} \cos \left(\delta_{f}-\alpha_{f}\right)=v \cos \beta, \\
& v_{w, r} \sin \alpha_{r}=l_{r} \cdot \dot{\psi}-v \sin \beta, \quad v_{w, r} \cos \alpha_{r}=v \cos \beta .
\end{aligned}
$$

In stable driving conditions, the tire side slip angle $\alpha_{i}$ is normally not larger than five degrees and the above equation can be simplified by substituting $\sin x \approx x$ and $\cos x \approx 1$. The classic equations for the tire side slip angles are then given as

$$
\alpha_{f}=-\beta+\delta_{f}-\frac{l_{f} \cdot \dot{\psi}}{v}, \quad \alpha_{r}=-\beta+\frac{l_{r} \cdot \dot{\psi}}{v} .
$$

The equations (18)-(22) can be expressed in the state space representation form. The system states are the side slip angle of the sprung mass $\beta$, the yaw rate $\dot{\psi}$, the roll angle $\phi$, the roll rate $\dot{\phi}$, the roll angle of the unsprung mass at the front axle $\phi_{t, f}$ and at the rear axle $\phi_{t, r}$. Let the state vector be the following:

$$
x_{r}=\left[\begin{array}{llllll}
\beta & \dot{\psi} & \phi & \dot{\phi} & \phi_{t, f} & \phi_{t, r}
\end{array}\right]^{T} .
$$

Using the state vector, the differential algebraic model defined by Equations (18)-(22) is transformed into a state space representation form:

$$
\dot{x}_{r}=A_{r}\left(\rho_{r}\right) x_{r}+B_{1 r_{v}}\left(\rho_{r}\right) d_{r}+B_{2 r_{v}}\left(\rho_{r}\right) u_{r} .
$$

The disturbance is the front wheel steering angle: $d_{r}=\delta_{f}$, while the control inputs are set to be:

$$
u_{r}=\left[\begin{array}{lll}
\Delta F_{b} & u_{a f} & u_{a r}
\end{array}\right]^{T}
$$


In this approach of the rollover problem the active suspensions generate two stabilizing roll moments at the front and the rear, which can be considered as the effects of the suspension forces

$$
u_{a f}=\left(f_{f l}-f_{f r}\right) \ell_{w}, \quad u_{a r}=\left(f_{r l}-f_{r r}\right) \ell_{w} .
$$

The roll moments required are distinguished equally at the suspension components:

$$
\begin{gathered}
f_{f l}=\frac{u_{a f}}{2 l_{w}}, \quad f_{f r}=-\frac{u_{a f}}{2 l_{w}}, \\
f_{r l}=\frac{u_{a r}}{2 l_{w}},
\end{gathered}
$$

The third control input is the difference in brake forces between the left and right-hand sides of the vehicle:

$$
\Delta F_{b}=\left(F_{b r l}+d_{2} F_{b f l}\right)-\left(F_{b r r}+d_{1} F_{b f r}\right),
$$

where $d_{1}$ and $d_{2}$ are distances, which depend on the steering angle. In the implementation of the controller means that the control action be distributed at the front and the rear wheels at either of the two sides.

The differential equations depend on the forward velocity $v$ and the adhesion coefficient $\mu$ of the vehicle nonlinearly. It is assumed that the forward velocity and the adhesion coefficient are available, i.e. these parameters are estimated on-line by using the on-board sensors. A grey-box identification method based on an observer design was proposed in Gáspár et al. (2006). The scheduling vector $\rho_{r}$ is selected with four scheduling variables

$$
\rho_{r}=\left[\begin{array}{llll}
\rho_{1} & \rho_{2} & \rho_{3} & \rho_{4}
\end{array}\right]
$$

with $\rho_{1}=\mu, \rho_{2}=\frac{\mu}{v}, \rho_{3}=\frac{\mu}{v^{2}}$ and $\rho_{4}=\frac{1}{v}$.

\section{Supervisory global control}

The control design for suspension system and rollover prevention is performed on a fullcar vehicle model. When a fault occurs in the active suspension system, its role is assumed by the active brake.The orchestration of the two independent subsystems, i.e. the suspension subsystem and active brake, respectively, should be solved by a dedicated mechanism in order to guarantee a desired level of the required performance.

The detection of an imminent rollover is based on the monitoring of the lateral load transfers for both axles. The lateral load transfer can be given:

$$
\Delta F_{z, i}=\frac{k_{t, i} \phi_{t, i}}{l_{w}}
$$

where $i$ denotes the front and rear axles. They can be normalized in such a way that the load transfer is divided by the total axle load:

$$
R_{i}=\frac{\Delta F_{z, i}}{F_{z, i}}
$$

The normalized load transfer $R_{i}$ value corresponds to the largest possible load transfer. If the $R_{i}$ takes on the value \pm 1 then the inner wheels in the bend lift off. The limit cornering 
condition occurs when the load on the inside wheels has dropped to zero and all the load has been transferred onto the outside wheels. Let

$$
R=\max \left\{R_{f}, R_{r}\right\} .
$$

The roll angles of the unsprung masses have an important role in the monitoring of rollovers, since the calculation of the normalized load transfers is based on these signals.

In the control design both the rollover and the suspension problems are taken into consideration. In this combined structure a new weighting strategy is proposed in order to meet several performance demands, such as enhancing passenger comfort, increasing rollover stability and road holding, guaranteing suspension working space and reducing energy consumption. In the rollover problem the performance outputs for control design are the lateral acceleration, the lateral load transfers at the front and the rear, and the control inputs:

$$
z_{r}=\left[\begin{array}{llll}
a_{y} & \Delta F_{z, f} & \Delta F_{z, r} & u_{r}
\end{array}\right]^{T} .
$$

In the suspension problem the performance outputs

$$
z_{s}=\left[\begin{array}{llll}
a_{z} & z_{s f} & z_{s r} & u_{s}
\end{array}\right]^{T}
$$

for control design are the passenger comfort (i.e. heave acceleration), the suspension deflections and the control inputs. The measured outputs are the lateral acceleration of the sprung mass, the derivative of the roll angle and the suspension deflections at the suspension components: $y_{r}=\left[\begin{array}{ll}a_{y} & \dot{\phi}\end{array}\right]^{T}$ and $y_{s}=\left[\begin{array}{ll}z_{s f} & z_{s r}\end{array}\right]^{T}$.

In order to achieve the desired reconfiguration of the redundant subsystems a straight solution would be to apply merely a switching strategy that would change between the two subsystems in emergency, i.e. when an imminent rollover occur. By applying a switching strategy based on a suitable threshold imposed for the value of the normalized load transfer $R$ (switching surface) would be a reliable solution for the design of the individual controllers. However, it would generate the problem of the transients during the switching instances, i.e. the required performance level should be imposed by special techniques in that case.

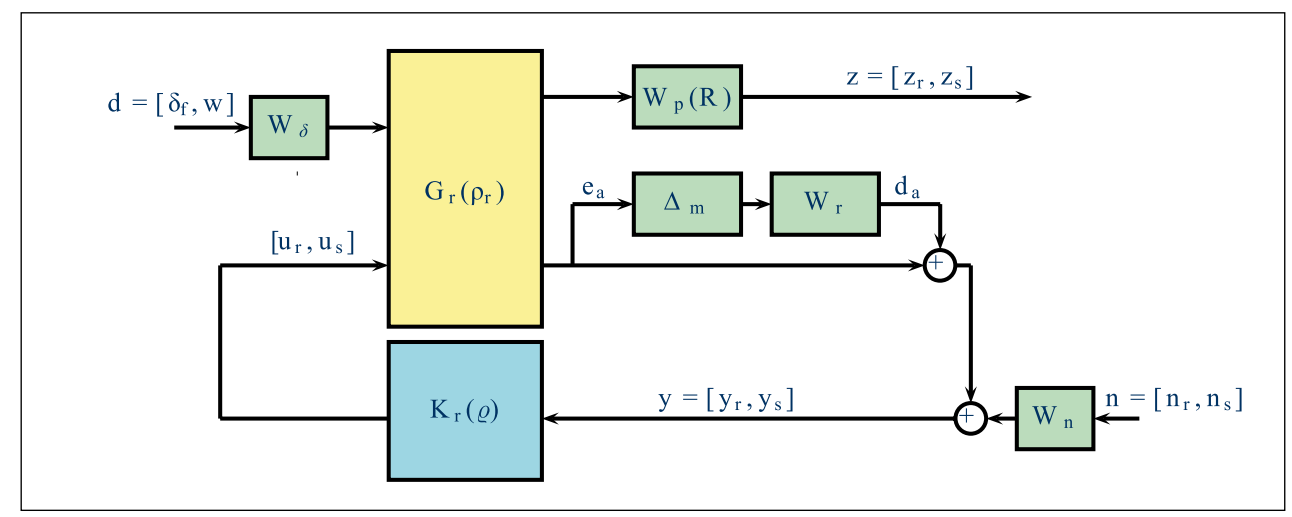

Fig. 3. The closed-loop interconnection structure for the control design

Instead of considering a switching surface the reconfiguration of the control structure is solved by an LPV strategy, which is presented in this section. The values of the the normalized load 
transfer $R$ are used as a scheduling variable for a LPV design where a guaranteed performance level during the reconfiguration is achieved through the design process - through a common Lyapunov function. This is possible by using a suitable weighting strategy where the critical parameter $R$ schedules the performance weight functions.

The closed-loop interconnection structure for rollover prevention and for suspension design are shown in Figure 3. The purpose of the weighting functions is to keep the lateral acceleration, the lateral load transfers, the heave acceleration, the suspension deflection and the control inputs small over the desired frequency range. The weighting functions chosen for performance outputs can be considered as penalty functions: they are selected large in a frequency range where small signals are desired, and small where larger performance outputs can be tolerated.

The weighting function for the lateral acceleration, for the heave acceleration and for the suspension deflection are selected in the following way:

$$
\begin{aligned}
& W_{p, a y}=\phi_{a y}(R) \frac{A_{1}\left(\frac{s}{T_{a}}+1\right)}{\left(\frac{s}{T_{b}}+1\right)}, \\
& W_{p, a z}=\frac{A_{2}\left(\frac{s}{T_{c}}+1\right)}{\left(\frac{s}{T_{d}}+1\right)}, \\
& W_{p, z s}=\frac{A_{3}\left(\frac{s}{T_{e}}+1\right)}{\left(\frac{s}{T_{f}}+1\right)},
\end{aligned}
$$

respectively, with time constants $T_{i}$ and proportional coefficients $A_{i}$. The weighting functions $W_{p, u s}$ and $W_{p, u r}$ for the control inputs guarantee the limitation of the control forces.

For safety the weighting function $W_{p, a y}$ for the lateral acceleration plays the most important role. The parameter-dependent gain $\phi_{a y}(R)$ in the weighting function $W_{p, a y}$ is selected as a function of parameter $R$ :

$$
\phi_{a y}(R)=\left\{\begin{array}{cll}
0 & \text { if } & |R|<R_{a} \\
\frac{\left(|R|-R_{a}\right)}{\left(R_{b}-R_{a}\right)} & \text { if } & R_{a} \leq|R| \leq R_{b} \\
1 & \text { if } & |R|>R_{b}
\end{array}\right.
$$

where $R_{a}, R_{b}$ are pre-defined and constants in fault-free case.

The gain $\phi_{a y}$ is increased in order to minimize the lateral acceleration and prevent the rollover of the vehicle. As the gain $\phi_{a y}$ increases the lateral acceleration decreases, since the active brake influences the lateral acceleration directly. $R_{a}$ defines the critical status when the vehicle is in an emergency. Parameter $R_{b}$ shows how fast the control should focus on minimizing the lateral acceleration. In the lower range of $R$ the gain $\phi_{a y}$ must be small, and in the upper range of $R$ the gains must be large. Consequently, the weighting functions must be selected in such a way that they minimize the lateral load transfers in emergency situations. However in normal cruising situations the control do not focus on the lateral load transfers since the weight is small.

The suspension system reduces the rollover risk when $|R| \geq R_{a}$. The suspension forces are modified by the fictitious forces coming from the stabilizing moments, see equation In an emergency, i.e. when $\left|\rho_{R}\right| \geq R_{s}$, the suspension system must reduce the rollover risk and guaranteeing passenger comfort (and pitch angle) is no longer a priority. The suspension forces are modified by the fictitious forces coming from the stabilizing moments, see equation 
(27). The forces at the front and the rear in both sides are the following:

$$
\begin{aligned}
& f_{f l, \text { new }}=f_{f l}+\frac{u_{r f}}{2 l_{w}}, \quad f_{f r, \text { new }}=f_{f r}-\frac{u_{r f}}{2 l_{w}}, \\
& f_{r l, \text { new }}=f_{r l}+\frac{u_{r r}}{2 l_{w}}, \quad f_{r r, \text { new }}=f_{r r}-\frac{u_{r r}}{2 l_{w}} .
\end{aligned}
$$

In the event of a fault the range of the operation of the brake system must be extended and the wheels are decelerated gradually rather than rapidly if the normalized load transfer has reached its critical value. A small value of $R_{a}$ corresponds to activating the brake system early and gradually, whereas a large value of $R_{a}$ corresponds to activating the brake system rapidly. Thus, the design parameter $R_{a}$ is chosen to be scheduled on fault information $\rho_{f}$.

$$
R_{a, \text { new }}=R_{a}-\frac{\rho_{f}}{\alpha}
$$

where $\rho_{f}$ is the normalized value of the fault information and $\alpha$ is a constant factor.

Introducing the health information about the suspension subsystem (through the fault signal $\rho_{f}$ ) in the control design has the benefit in reducing the brake actuation during operational time. This is achieved by a varying threshold of the activation level, which is $R_{a}$ for the fault-free case and it decreases only as an exception when a loss of effectiveness/fault occur in contrast to the conservative setting $R_{a}-\frac{1}{\alpha}$ when no information about the suspension is supposed to be available.

The uncertainties of the nominal model are represented by the weighting function $W_{r}$ in such a way that in the low frequency domain the uncertainties are about $10 \%$ and in the upper frequency domain they are up to $100 \%$. The input scaling weights $W_{\delta}$ and $W_{w}$ normalize the disturbances to the maximum expected command. $W_{n, a y}, W_{n, \dot{\phi}}, W_{n, \dot{\psi}}$ and $W_{n, s i j}$ take into account the sensor noises in the control design.

In order to describe the control objective, the parameter dependent augmented plant $P(\varrho)$ must be built up using the closed-loop interconnection structure. The augmented plant $P(\varrho)$ includes the parameter dependent vehicle dynamics and the weighting functions.

$$
\left[\begin{array}{c}
\tilde{z} \\
\hline y
\end{array}\right]=\left[\begin{array}{l|l}
P_{11}(\varrho) & P_{12}(\varrho) \\
\hline P_{21}(\varrho) & P_{22}(\varrho)
\end{array}\right]\left[\begin{array}{c}
w \\
\hline u
\end{array}\right],
$$

where $w=\left[\begin{array}{lll}d & n & d_{m}\end{array}\right]$ and $\tilde{z}=\left[\begin{array}{ll}z & e_{m}\end{array}\right]$. The signals $d_{m}, e_{m}$ are the output of the uncertainty block $\Delta_{m}$ and its input, respectively. $\varrho$ contains both the scheduling variables from the vehicle modeling and the monitoring variables. The closed-loop system $M(\varrho)$ is given by a lower linear fractional transformation (LFT) structure:

$$
M(\varrho)=\mathcal{F}_{\ell}(P(\varrho), K(\varrho)),
$$

where $K(\varrho)$ depends on the scheduling parameter $\varrho$. The purpose of the control design is to robustly minimize the induced $\mathcal{L}_{2}$ norm of a LPV system $M(\varrho)$ with zero initial conditions, which is given by

$$
\inf _{K} \sup _{\varrho \in \mathcal{F}_{\mathcal{P}}\|w\|_{2} \neq 0, w \in \mathcal{L}_{2}} \sup _{\|w\|_{2}} \frac{\|\tilde{z}\|_{2}}{\|w\|^{2}}
$$


The solution of an LPV problem is based on the set of infinite dimensional LMIs being satisfied for all $\rho \in \mathcal{F}_{\mathcal{P}}$, thus it is a convex problem, Rough \& Shamma (2000); Wu (2001). In practice, this problem is set up by gridding the parameter space and solving the set of LMIs that hold on the subset of $\mathcal{F}_{\mathcal{P}}$, see Packard \& Balas (1997). The LPV control is constructed by the Parameter Dependent Lyapunov Functions (PDLF) in which the conservatism of the control design is reduced.

To specify the scheduled performance weights for the LPV design the scheduling variables are defined through a lookup-table given on a suitable grid. The grid is determined by $v, \mu, R$ and $\rho_{f}$ as follows: $v=[20, \ldots, 120] \mathrm{kph}, \mu=[0.1, \ldots, 1.1]$ and $R=\left[0, R_{a}, R_{b}, 1\right]$. The scheduling parameter $\rho_{f}$, which is the fault information provided by the FDI filter, can be taken from interval $\rho_{f}=[0,1]$. The zero value of $\rho_{f}$ corresponds to the non-faulty operation and the value 1 to the full hydraulic actuator failure. The gridding reflects the qualitative changes of the performance weights, i.e. the scheduling variables. The robust stability and performance are guaranteed by the LPV design process, see Packard \& Balas (1997); Wu (1995).

\section{Design of tracking control}

The starting point for the tracking control design of the active suspension actuator is a quarter car LPV model of the suspension system augmented with a nonlinear actuator dynamics. The actuator is a nonlinear switched system (bimodal system) where the switch is triggered by the sign of the damper velocity.

In Figure 4 a two-degree-of-freedom quarter-car model is shown. The body mass $m_{S}$ represents the sprung mass, which corresponds to one of the corners of the vehicle, and the unsprung mass $m_{u}$ represents the wheel at one corner. The parameters $k_{t}, k_{s}, b_{s}$ are the tyre stiffness, the suspension stiffness, and the damping rate of the suspension, respectively. The control signal $F$ is generated by the actuator. The disturbance $d$ is caused by road irregularities.

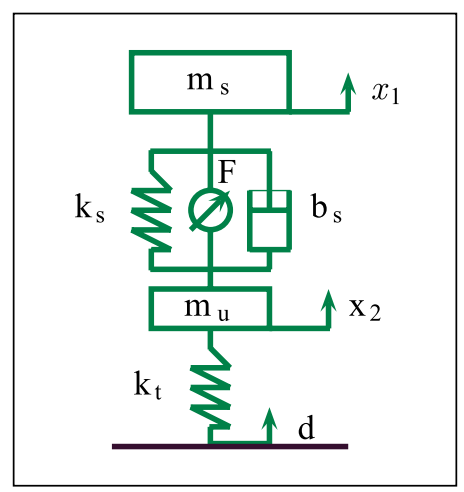

Fig. 4. Quarter-car model

The force equations of the quarter-car model are:

$$
\begin{aligned}
& F_{m_{s}}=F_{k}+F_{b}-F, \\
& F_{m_{u}}=-F_{k}-F_{b}-F_{t}+F .
\end{aligned}
$$


The suspension damping force $F_{b}$ is given by (9), the suspension spring force $F_{k}$ is defined by (10) while the tire force $F_{t}$ is given by (11) and $F$ is the force of the actuator, respectively.

The state space representation of the quarter-car model can be formalized with the state vector $x=\left[\begin{array}{llll}x_{1} & x_{2} & x_{3} & x_{4}\end{array}\right]^{T}$, where $x_{1}$ and $x_{2}$ denote the vertical displacement of the sprung mass and the unsprung mass, respectively, and $x_{3}, x_{4}$ denote their derivatives.

$$
\begin{aligned}
& \dot{x}_{3}=\frac{1}{m_{s}}\left(r_{k}\left(x_{2}-x_{1}\right)+r_{b}\left(x_{4}-x_{3}\right)+b_{s}^{n l} \rho_{b} \sqrt{\rho_{b}\left(x_{4}-x_{3}\right)}-F\right), \\
& \dot{x}_{4}=\frac{1}{m_{u}}\left(-r_{k}\left(x_{2}-x_{1}\right)-r_{b}\left(x_{4}-x_{3}\right)-k_{t}\left(x_{2}-d\right)-b_{s}^{n l} \rho_{b} \sqrt{\rho_{b}\left(x_{4}-x_{3}\right)}+F\right),
\end{aligned}
$$

where $r_{b}=b_{s}^{l}-b_{s}^{s y m} \rho_{b}$ and $r_{k}=k_{s}^{l}+k_{s}^{n l} \rho_{k}$. Here $\rho_{b}=\operatorname{sgn}\left(x_{4}-x_{3}\right)$ and $\rho_{k}=\left(x_{2}-x_{1}\right)^{2}$ are selected as scheduling variables.

An active actuator which generates the necessary force for the suspension system is a fourway valve-piston system, in general. Denoting by $z$ the relative velocity one has $F=A_{P} P_{L}$, where $A_{P}$ is the area of the piston and $P_{L}$ is the pressure drop across the piston with respect to the front and rear suspensions. The derivative of $P_{L}$ is given by

$$
\dot{P}_{L}=-\beta P_{L}+\alpha A_{P} z+\gamma Q,
$$

in which $Q=Q_{0} x_{v}$ is the hydraulic load flow (with the notation $Q_{0}=\operatorname{sgn}(r) \sqrt{|r|}$ and $r=P_{S}-$ $\operatorname{sgn}\left(x_{v}\right) P_{L}$, moreover, $\alpha, \beta, \gamma$ are constants, $P_{S}$ is the supply pressure and $x_{v}$ is the displacement of the spool valve. The cylinder velocity acts as a coupling from the position output of the cylinder to the pressure differential across the piston. It is considered a feedback term, which has been analyzed by Alleyne \& Liu (2000).

The displacement of the spool valve is controlled by the input to the servo-valve $u$ :

$$
\dot{x}_{v}=\frac{1}{\tau}\left(-x_{v}+u\right),
$$

where $\tau$ is a time constant. Let $x_{5}$ and $x_{6}$ denote $P_{L}$ and $x_{v}$, respectively. Then, the actuator model can be written separately as

$$
\begin{aligned}
& \dot{x}_{5}=-\beta x_{5}+\alpha A_{P} z+\gamma Q_{0}\left(x_{5}, x_{6}\right) x_{6}, \\
& \dot{x}_{6}=-\frac{1}{\tau} x_{6}+\frac{1}{\tau} u_{a} .
\end{aligned}
$$

For both actuators it is hard to provide directly the command signals due to the high nonlinearities of these subsystems. Usually the controllers provide a force demand and in a second, postprocessing step the actual actuator commands are derived, see Alleyne \& Hedrick (1995). The tracking control algorithm is derived by using a backstepping method applied for each of the modes.

In order to show the principle of the backstepping method the notations of van der Schaft (2000) are used. The model of the whole suspension and actuator system with zero disturbance are written in the following form

$$
\begin{aligned}
\dot{\zeta} & =A \zeta+B \xi_{1}, \\
\dot{\xi}_{1} & =a_{1}\left(\zeta_{,} \xi_{1}\right)+b_{1}\left(\xi_{1}\right) \xi_{2}, \\
\dot{\xi}_{2} & =a_{2}\left(\xi_{2}\right)+b_{2} u,
\end{aligned}
$$


where $\zeta$ is the state vector of the quarter-car suspension model, $\xi_{1}$ and $\xi_{2}$ are the state variables of the actuator dynamics and furthermore

$$
\begin{aligned}
a_{1}\left(\zeta, \xi_{1}\right) & =-\beta \xi_{1}+\alpha A_{P} z, \\
b_{1}\left(\xi_{1}\right) & =\left\{\begin{array}{ll}
\gamma \sqrt{P_{S}-\xi_{1}}, & \xi_{2} \geq 0 \\
\gamma \sqrt{P_{S}+\xi_{1}}, & \xi_{2}<0
\end{array},\right. \\
a_{2}\left(\xi_{2}\right) & =-\frac{1}{\tau} \xi_{2}, \quad b_{2}=\frac{1}{\tau} .
\end{aligned}
$$

Let us assume that there exists a smooth feedback function $K(\zeta)$ (possibly in LPV form) such that the closed loop system

$$
\dot{\zeta}=A \zeta+B K(\zeta)
$$

is asymptotically stable with control Lyapunov function $V(\zeta)$. The dynamics of (53) corresponds to the high-level suspension system (13). Therefore, the feedback function $K(\zeta)$ corresponds to the pressure demand required by the high-level control, i.e. $K(\zeta)=P_{L, d e m}=\xi_{1, \text { dem }}$. In what follows $\xi_{2, \text { dem }}=\xi_{v \text {, dem }}$ denotes the demand of the spool valve displacement.

The backstepping design for the actuator subsystem can be performed in two steps. In the first step, let us consider $\xi_{2, d e m}$ as a virtual input and $y_{1}=\xi_{1}-K(\zeta)$ as a virtual output. Since $\xi_{1}$ is not a manipulable input, we would like to construct a feedback that guarantees the tracking of $K(\zeta)$ with $\xi_{1}$. It is reasonable therefore to define the tracking error to be linear and stable, i.e., $\dot{y}_{1}=-k_{1} y_{1}, k_{1}>0$. Using (53)-(54) the desired time-function for $\xi_{2, \text { dem }}$ can be computed as a nonlinear feedback of the form

$$
\xi_{2, \text { dem }}=\frac{1}{b_{1}\left(\xi_{1}\right)}\left[-a_{1}\left(\zeta, \xi_{1}\right)+\dot{K}(\zeta)-k_{1}\left(\xi_{1}-K(\zeta)\right)\right] .
$$

In the second step, the desired input is $u$ while the (virtual) output is defined as $y_{2}=\xi_{2}-$ $\xi_{2, \mathrm{dem}}$. For the tracking error, a stable linear dynamics is prescribed: $\dot{y}_{2}=-k_{2} y_{2}, k_{2}>0$. Using (53)-(55), we can now express the physically manipulable actuator input $u$ as a function of $\zeta$, $\xi_{1}$ and $\xi_{2}$ in the following form

$$
u=\frac{1}{b_{2}}\left[-a_{2}\left(\xi_{2}\right)+\dot{\xi}_{2, d e m}\right]
$$

By applying the above design, the closed loop system will be asymptotically stable with control Lyapunov function $S(\zeta)=V(\zeta)+\frac{1}{2} y_{1}^{2}+\frac{1}{2} y_{2}^{2}$ Sepulchre et al. (1997). It is important to note that the obtained feedback law (58) is a state-dependent switching function because of the switching term $b_{1}\left(\xi_{1}\right)$ (see (56)) and it will be shown later that $\xi_{2, \text { dem }}$ can be approximated by a smooth function without affecting the validity of the method.

Since the actual feedback law generated by the LPV controller is a rather complicated function of the state variables, and we do not know the road excitation disturbances in advance, the above controller design procedure cannot be implemented in its original theoretical form. Therefore in what follows we will consider a more realistic assumption, when the reference for $\xi_{1}$ is computed by the high-level LPV controller, and for the trajectory tracking the time derivatives of the reference signals are computed numerically.

The reference for the pressure $x_{5}$, which is is denoted by $x_{5, \text { dem }}$, is computed by the high level LPV controller and for the trajectory tracking the time derivatives of the reference signals are computed numerically. The required tracking error dynamics is defined as

$$
\dot{x}_{5}-\dot{x}_{5, \mathrm{dem}}=-k_{1}\left(x_{5}-x_{5, \mathrm{dem}}\right)
$$


with a chosen positive constant parameter $k_{1}$.

Then the reference $x_{6, \text { dem }}$ is given by

$$
x_{6, \mathrm{dem}} \gamma \sqrt{P_{S}-\operatorname{sgn}\left(x_{6, \mathrm{dem}}\right) x_{5}}=\Psi\left(x_{5}, x_{5, \mathrm{dem}}, z\right),
$$

i.e.

$$
x_{6, \mathrm{dem}}= \begin{cases}\frac{\Psi\left(x_{5}, x_{5, \mathrm{dem}}, z\right)}{\gamma \sqrt{P_{S}-x_{5}}}, & x_{6, \mathrm{dem}} \geq 0 \\ \frac{\Psi\left(x_{5}, x_{5, \mathrm{dem}}, z\right)}{\gamma \sqrt{P_{S}+x_{5}}} & x_{6, \mathrm{dem}}<0\end{cases}
$$

with the notation $\Psi\left(x_{5, \text { dem }}, z\right)=\beta x_{5}-\alpha A_{p} z+\dot{x}_{5, \text { dem }}-k_{1}\left(x_{5}-x_{5, \text { dem }}\right)$. Note that while $\Psi$ is a smooth function, by taking the time derivative of both sides of (60) one has

$$
\dot{x}_{6, \mathrm{dem}}= \begin{cases}\frac{\dot{\Psi}\left(x_{5, \mathrm{dem}} z\right)}{\gamma \sqrt{P_{S}-x_{5}}}+\frac{\dot{x}_{5, \mathrm{dem}} x_{6, \mathrm{dem}}}{2 \gamma\left|P_{S}-x_{5}\right|}, & x_{6, \mathrm{dem}}>0 \\ \frac{\Psi\left(x_{5, \mathrm{dem},}, z\right)}{\gamma \sqrt{P_{S}+x_{5}}}-\frac{\dot{x}_{5, \mathrm{dem}} x_{6, \mathrm{dem}}}{2 \gamma\left|P_{S}+x_{5}\right|} & x_{6, \mathrm{dem}}<0\end{cases}
$$

i.e., $x_{6, \mathrm{dem}}$ in general is not smooth - it is continuous but not differentiable. However it can be arbitrary approximated by a smooth function $\bar{x}_{6, \mathrm{dem}}$ for which tacking the tracking error dynamics

$$
\dot{x}_{6}-\dot{\bar{x}}_{6, \mathrm{dem}}=-k_{2}\left(x_{6}-\bar{x}_{6, \mathrm{dem}}\right) .
$$

with a chosen positive constant parameter $k_{2}$ is meaningful. Moreover this $\bar{x}_{6, d e m}$ can be chosen such that $\bar{x}_{6, \mathrm{dem}} \neq x_{6, \mathrm{dem}}$ only on a small neighborhood of the origin (the discontinuity point of $\left.\dot{x}_{6, \mathrm{dem}}\right)$, e.g. by taking an expression on $|\Psi| \leq \epsilon$ linear in $\Psi$, i.e. $\bar{x}_{6, \mathrm{dem}}=\beta \Psi$ with a suitable $\beta$. Since $x_{6, d e m}$ should satisfy (60) this choice does not affect the values of the desired $x_{5}$ considerably.

Finally, the following expression for the physical input is deduced:

$$
u_{a}=x_{6}+\tau \dot{\bar{x}}_{6, \mathrm{dem}}-\tau k_{2}\left(x_{6}-x_{6, \mathrm{dem}}\right) .
$$

In order to practically implement the control law, we need to compute the time derivatives of $x_{5, \mathrm{dem}}$ and $x_{6, \mathrm{dem}}$, which can be done in a number of ways depending on the measurement noise conditions and the required precision, for details see Gáspár et al. (2008).

\section{Design of the FDI filter}

Significant research results have been published for the general FDI problem and several methods have been proposed, e.g. the parity space approach, the multiple model method, detection filter design using a geometric approach, or the dynamic inversion based detection, see Massoumnia (1986); Gertler (1997); Szigeti et al. (2001). Most of the design approaches refer to linear, time-invariant (LTI) systems, but references to some nonlinear cases are also found in the literature, see Stoustrup \& Niemann (1998); Chen \& Patton (1999). An $\mathcal{H}_{\infty}$ approach to design a fault detection and isolation gain-scheduled filter for $L P V$ systems was presented by Abdalla et al. (2001); Bokor \& Balas (2004). There are also numerous papers dealing with the design of reconfigurable controls, which include the design of FDI filters, the design of reconfigurable controllers and the design of reconfiguration mechanisms. Applications of reconfigurable control systems are found in different fields, see e.g. Fischer \& Isermann (2004); Kanev \& Verhaegen (2000). 
Possible faults of the actuators (loss of effectiveness) can be detected by reconstructing the actual suspension forces. Having measured the signals $y_{1}=\dot{x}_{3}, y_{2}=\dot{x}_{4}$ and $y_{3}=x_{2}-x_{1}$ an inversion based detection filter is proposed, Balas et al. (2004); Szabó et al. (2003). In the construction of the filter the first step is to express $F$ from (48) and in these expression we plug in the known values $y_{i}$ :

$$
F=|z|+b_{s}^{n l} \rho_{b} \sqrt{|z|}+r_{k} y_{3}-m_{s} y_{1} .
$$

In this expression the value of the relative velocity $z$ is not measured. The road disturbance is an unknown input signal but from the equations (48), (49) one has

$$
m_{s} \dot{x}_{3}+m_{u} \dot{x}_{4}=-k_{t}\left(x_{2}-d\right) \text {. }
$$

By plugging back the obtained expressions in the original equations one has the system $\dot{x}_{3}$ $=\frac{r_{k}}{m_{s}}\left(x_{2}-x_{1}\right)-\frac{r_{k}}{m_{s}} y_{3}+y_{1}$ and $\dot{x}_{4}=-\frac{r_{k}}{m_{u}}\left(x_{2}-x_{1}\right)+\frac{r_{k}}{m_{u}} y_{3}+y_{2}$, where the relative velocity is not measured. The resulting LPV system

$$
\dot{z}=-r_{k} m_{e} z+r_{k} m_{e} y_{3}+y_{2}-y_{1}
$$

with $m_{e}=\frac{m_{u}+m_{s}}{m_{u} m_{s}}$ will be observable.

For active actuators, since the real actuators might present a saturation effect, in addition to compare the reconstructed forces with the force demands provided by the robust LPV controllers it is necessary to check, if the actual forces are lower then those corresponding to the saturation level of the actuators.

To obtain the final fault detection filter equations (51) and (52) are used as:

$$
\begin{aligned}
& \dot{\tilde{x}}_{5}=-\beta \tilde{x}_{5}+\alpha A_{P} \hat{z}+\gamma Q_{0, n o m}(\hat{F}) \tilde{x}_{6}, \\
& \dot{\tilde{x}}_{6}=-\frac{1}{\tau_{\text {nom }}} \tilde{x}_{6}+\frac{1}{\tau_{\text {nom }}} u_{a},
\end{aligned}
$$

where $\hat{z}$ and $\hat{F}$ are the estimated damper velocity and damper force values, respectively. A possible actuator fault affects the terms $Q_{0}$ through a modified value of $P_{S}$ and the time constant $\tau$, respectively. The nominal values of these parameters (i.e. for the fault free case) are denoted by the subscript nom.

For the fault free case one should have $e_{5}=x_{5}-\tilde{x}_{5} \approx 0$ and $e_{6}=x_{6}-\tilde{x}_{6} \approx 0$, respectively. Since the initial conditions are not known, an observer need to be constructed for (65) and (66), (67) respectively, to test these conditions. For a Leuenberger-type observer a design method for bimodal systems was reported in Juloski et al. (2007). For this case, however, the nonsmooth term $Q_{0}$ - which in turn makes the system to be bimodal - is considered as scheduling variable. Hence a more conventional LPV observer can be constructed.

For a LPV system that depends affinely on the scheduling variables an LPV observer gain can be designed using LMI techniques: let us recall that an LPV system is said to be quadratically stable if there exist a matrix $P=P^{T}>0$ such that $A(\rho)^{T} P+P A(\rho)<0$ for all the parameters $\rho$. A necessary and sufficient condition for a system to be quadratically stable is that this condition holds for all the corner points of the parameter space, i.e., one can obtain a finite system of linear matrix inequalities ( $L M I s$ ) that have to be fulfilled for $A(\rho)$ with a suitable positive definite matrix $P$, see Gahinet (1996).

In order to obtain a quadratically stable observer the $L M I$

$$
A_{o}^{T}(\rho) P+P A_{o}(\rho)<0
$$


must hold for suitable $K(\rho)$ and $P=P^{T}>0$, with $A_{o}=A+K C$. By introducing the auxiliary variable $L(\rho)=P K(\rho)$, one has to solve the following set of LMIs on the corner points of the parameter space:

$$
A(\rho)^{T} P+P A(\rho)-C^{T} L(\rho)^{T}-L(\rho) C<0 .
$$

By solving these LMIs a suitable observer gain is obtained:

$$
K(\rho)=P^{-1} L(\rho) .
$$

If $e=\left\|e_{5}\right\|_{2}+\left\|e_{6}\right\|_{2}$ is greater than a given threshold, then a fault must be present in the system and a fault signal is emitted to the higher level controller, used in the controller reconfiguration process. The threshold level influences the fault-detection delay, i.e. high threshold level corresponds to increased delay. However, due to disturbances, sensor noises and the modeling uncertainties this level cannot be arbitrarily small and it is determined using engineering knowledge.

\section{Simulation example}

In this section the operation of the integrated control is presented and analyzed through simulation examples.

In the first example the operation of the two-level controller is demonstrated. The controller, which combines a high-level LPV controller and a low-level nonlinear controller, is built in Matlab/Simulink software environment.

In the simulation example an upper-level controller is designed based on the LPV method, which generates a required control force. The controlled systems are tested on a bad-quality road, on which bumps of four different heights disturb the motion of the vehicle: the height of the bumps are $6 \mathrm{~cm}, 4 \mathrm{~cm}, 2 \mathrm{~cm}$ and $4 \mathrm{~cm}$, respectively. Between the bumps there are velocitydependent stochastic road excitations. The time responses of the road excitation, the heave acceleration, the relative displacement and the control force in the front and left-hand-side are illustrated in Figure 5. The bumps with extremely large amplitude cause large acceleration of the sprung mass and large relative displacement between the two masses.

Thanks to the controller the effects of the road disturbances on the performances are acceptable since the values of the performance signals tend to zero in a short time period. The suspension problem is solved by the force defined by the controller in the upper-level.

Then the low-level controller is applied in order to track the designed force. The operation of the force-tracking controller based on the backstepping method is illustrated in Figure 6. In the control design the parameters are selected as $k_{1}=20$ and $k_{2}=20$. In the simulation example it is assumed that the sampling time of the measured signals is selected $T_{S}=0.01 \mathrm{sec}$, which corresponds to practice.

The illustrated signals are the pressure drop across the piston, the displacement of the spool valve, the control input, the achieved force and the RMS of the force error. The achieved force generated by the actuator tends to the required force. The RMS of the force error, see Figure 7, shows that the generated force approximates the required force with high precision.

The second example illustrates the operation of the FDI filter applied to an active suspension system. The dashed red line presents the required force designed by the control system. The current force must be calculated by using the measured signals. A filter is used to calculate the current force by using an inversion method and the measured signals, i.e. the accelerations 


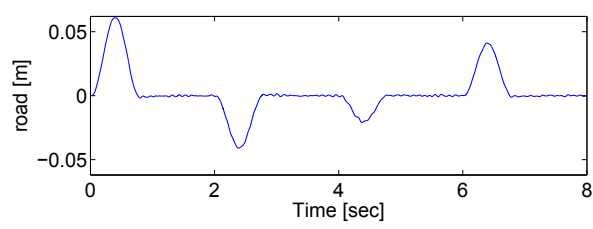

(a) Road excitation

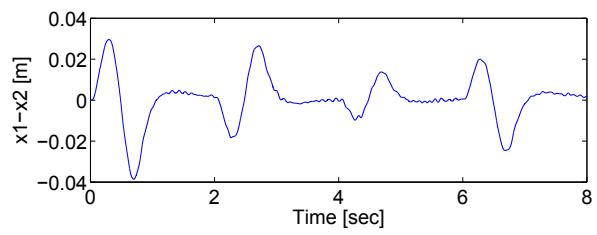

(c) Relative displacement

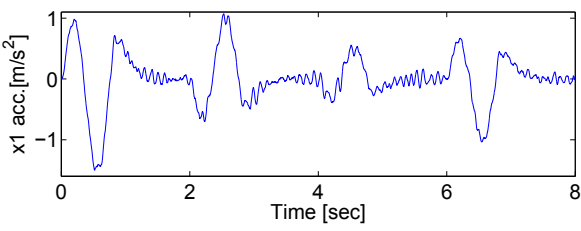

(b) Sprung mass acceleration

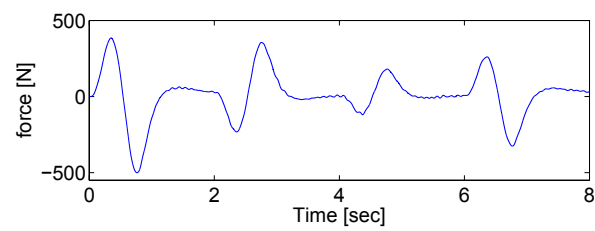

(d) Force required by upper level controller

Fig. 5. Control input required by the upper-level controller

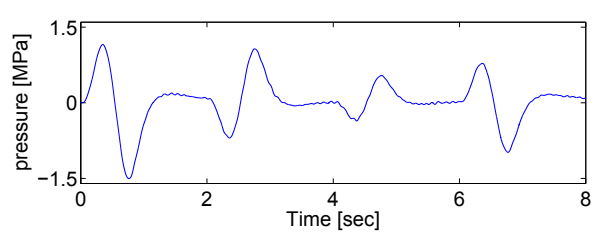

(a) Pressure

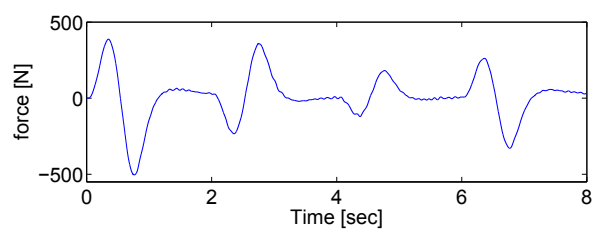

(c) Force generated by low level controller

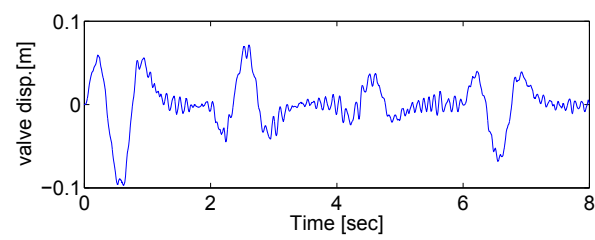

(b) Valve displacement

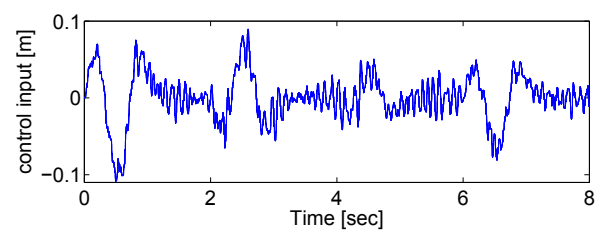

(d) Control input

Fig. 6. Analysis of the tracking properties using the backstepping method

of the sprung mass and the unsprung mass, and the relative displacement between the two masses.

The reconstructed force is illustrated by the solid blue line in the upper plot of Figure 8 . The force is compared with the force produced by a fault free suspension system (dashed line). The FDI filter also gives the signals depicted in blue in the lower plot of Figure 8, while the red signal is the chosen threshold level expressed in a given percent of the desired force. Since the obtained error level will be greater than this threshold, a fault signal is emitted indicating a faulty actuator.

In the third example the operation of the fault-tolerant integrated control that uses the designed FDI filter is illustrated. The vehicle performs a cornering maneuver with $70 \mathrm{~km} / \mathrm{h}$. 


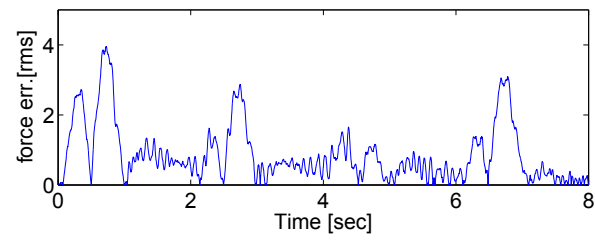

Fig. 7. Force error (RMS)
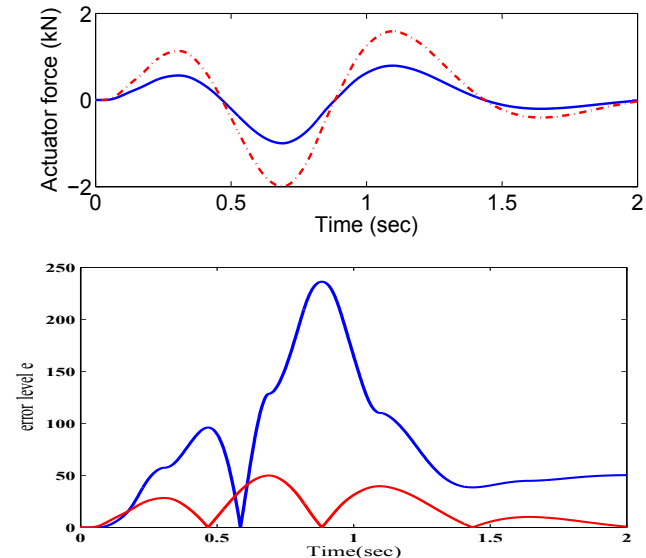

Fig. 8. The result of the FDI procedure

velocity. During the cornering maneuver the lateral acceleration increases and thus the roll angle of unsprung masses also increases. The time response of the steering angle, the lateral acceleration, the forward velocity and the normalized lateral load transfer at the rear are depicted in Figure 9.
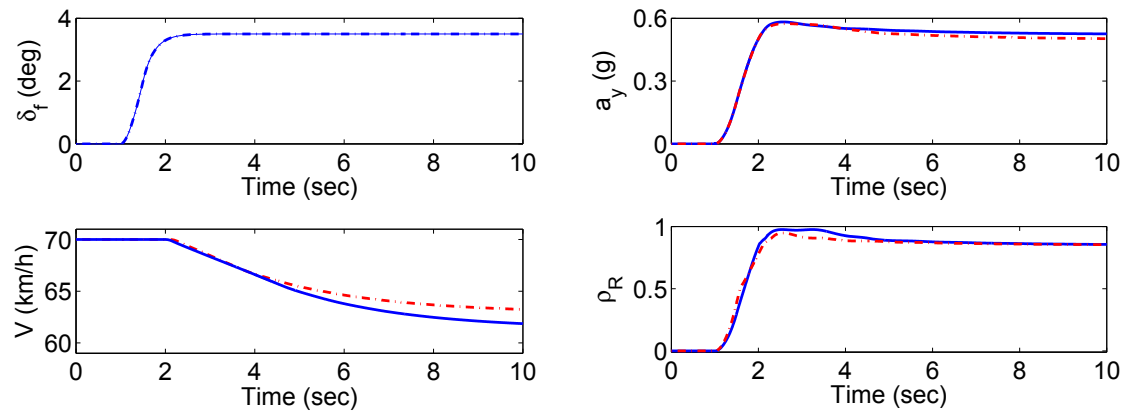

Fig. 9. Time responses of the control system

Since the monitoring scheduling variable, i.e., the normalized lateral load transfer $\rho_{R}$ increases the suspension system generate stabilizing roll moment to balance the overturning moment. However, the normalized lateral load transfer also exceeds the predefined critical value $R_{a}$ 
and the brake generated a force with which the direction of the vehicle slightly modified and consequently the effect of the lateral force reduces. Figure 10 shows the control signals, i.e. the braking force at the rear and all the suspension forces.

Then it is assumed that an actuator failure in the suspension system has already been detected at the front and right. The time response of the control signals are also depicted in Figure 10. The solid blue line illustrates the fault operation and the dashed red line illustrates the faultfree case.
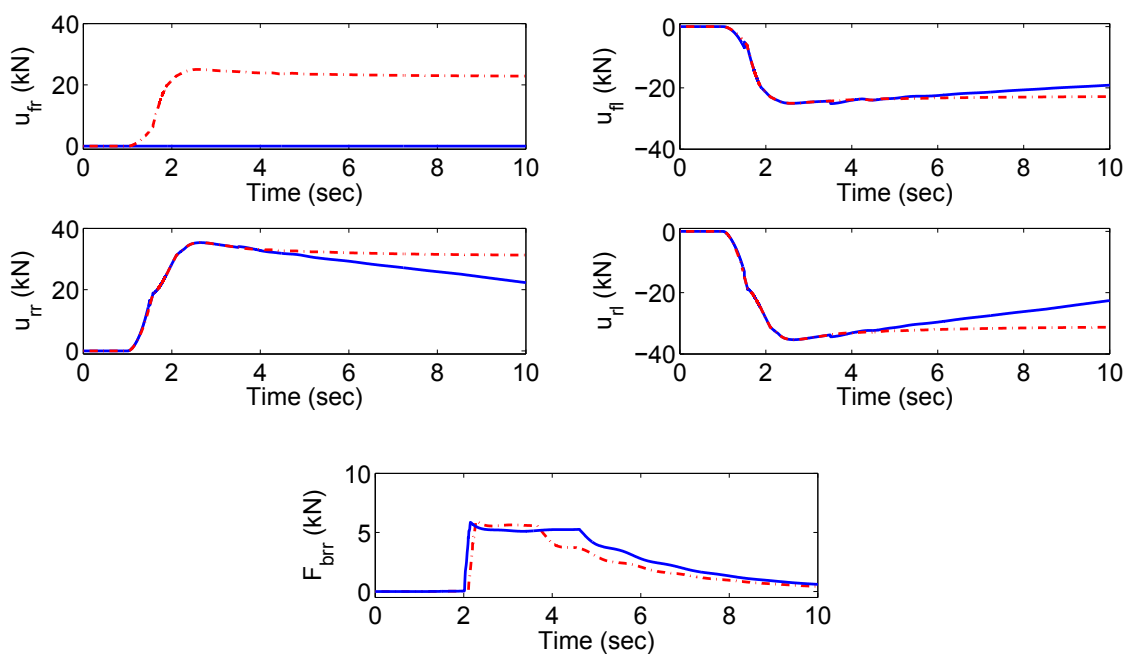

Fig. 10. Time responses of the control signals

It is observed that the normalized load transfer increases due to the reduced power of the actuators. According to the detected actuator fault the brake is activated at a smaller value of the critical normalized load transfer. Moreover, the duration of the required brake force is longer in the case of a suspension fault. Because of the braking action the suspension system generates the same forces (except in the fault component) as they are in the fault-free case.

In the fourth example the selection of $R_{a}$ and $R_{b}$ regarding the activation of the brake is critical. If the brake is activated at a large $R_{a}$ the probability of rollover increases. If the value $R_{a}$ was small, the brake would be activated very frequently. In case of a fault the selection of $\rho_{D}$ also has an important role. Finally, we shall examine the effects of varying the design parameter $R_{a}$ on the controlled system. In Figure 11 the peak lateral acceleration against forward velocity is plotted during a vehicle maneuver. $R_{b}$ is fixed at 0.95 and $R_{a}$ varies. The dash-dot, dashed and solid lines correspond to $R_{a}=0.7, R_{a}=0.8$ and $R_{a}=0.9$ respectively. With $R_{a}=0.7$ in the controlled system there is a gradual brake control, whereas when $R_{a}=0.9$, the brake system is not used until the normalized load transfer $\rho_{R}$ equals 0.9 , and the response of the yaw-roll model is the same as when only active suspensions are used. Thus the design parameters $R_{a}$ and $R_{b}$ can be used to shape the nonlinear response characteristics of the controlled system. In a non-faulty case, which means that suspension system is working well, it would be preferable to choose $R_{a}$ large. This corresponds to an active brake system that is not used for most of the time and activated very rapidly when the normalized load transfer exceeds the critical 


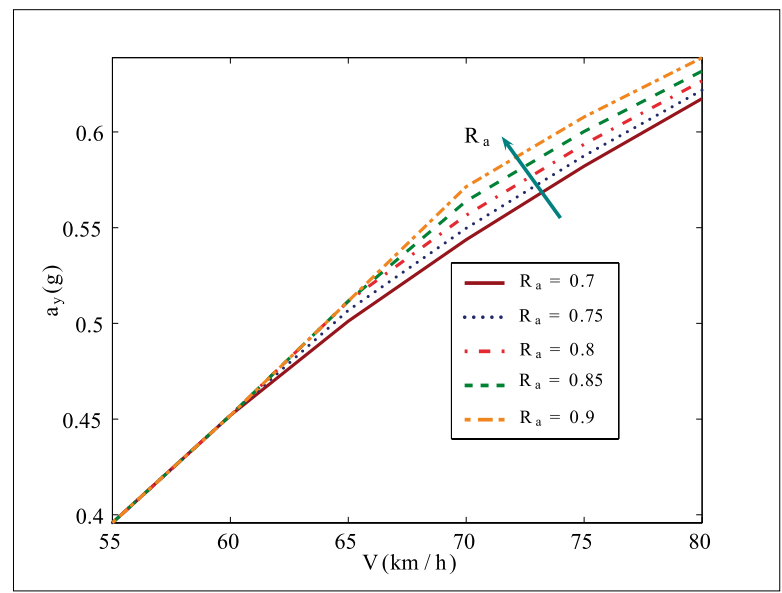

Fig. 11. Effect of parameter $R_{a}$ on lateral acceleration

value determined by $R_{a}$. However, this would result in a large lateral acceleration until the critical $R_{a}$ is reached. This would be a small price for the stability of roll motion. Because until the critical $R_{a}$ has been reached only the active suspensions, which do not affect directly the roll dynamics of the vehicle, are used.

On the other hand, if a hydraulic actuator fault occurs in the system it would be preferable to choose $R_{a}$ small. This corresponds to a combined control where the range of operation of the brake system is extended and the wheels are decelerated gradually rather than rapidly if the normalized load transfer has reached $R_{a}$. It is assumed that the actuator fault can occur as a loss of effectiveness, i.e. its power is reduced by some percent. It means that both control inputs are able to work simultaneously but the hydraulic actuator does not have maximum performance. It is a reasonable assumption in many cases because the occurrence of the failure indicates an effectiveness failure at an early stage. As a consequence, the design parameter $R_{a}$ can be chosen as a scheduling parameter based on the fault information.

\section{Conclusions}

In this paper an application of the Linear Parameter Varying method for the design of integrated vehicle control systems has been presented, in which several active components has been used in co-operation. In the control design besides performance specifications and uncertainties, the fault information has been taken into consideration. By monitoring suitable scheduling parameters in the LPV control, the reconfiguration of the control systems can be achieved, conflict between performance demands can be avoided and faults (loss in effectiveness) can be handled.

In the proposed scheme if a fault occurs in the active suspension system and it is detected by the FDI filter, the active brake assumes the role of the active suspension to enhance rollover prevention. A weighting strategy is applied in the closed-loop interconnection structure, in which the normalized lateral load transfer and the residual output of the FDI filter play an important role. A tracking controller and an FDI filter has been designed that provides the reference signal for the low-level actuator design and it also constitutes the supervisor con- 
troller for the reconfiguration. By using the LPV method the designed controller guarantees the desired stability and performance demands of the closed-loop system.

\section{Acknowledgements}

This work is supported by the Hungarian National Office for Research and Technology through grants TECH_08_2/2-2008-0088 is gratefully acknowledged.

The effort was sponsored by the Air Force Office of Scientific Research, Air Force Material Command, USAF, under the grant number FA8655-08-1-3016. The U.S Government is authorized to reproduce and distribute reprints for Governmental purpose notwithstanding any copyright notation thereon.

\section{References}

Abdalla, M., Nobrega, E. \& Grigoriadis, K. (2001). Fault detection and isolation filter design for linear parameter varying systems, Proceedings of the American Control Conference 2001, Vol. 5, IEEE, Arlington, VA, USA, pp. 3890-3895.

Alleyne, A. \& Hedrick, J. (1995). Nonlinear adaptive control of active suspensions, IEEE Transactions on Control Systems Technology 3(1): 94-101.

Alleyne, A. \& Liu, R. (2000). A simplified approach to force control for electro-hydraulic systems, Control Engineering Practice 8(12): 1347-1356.

Balas, G., Bokor, J. \& Szabó, Z. (2004). Tracking of continuous LPV systems using dynamic inversion, Proceedings of the 43rd IEEE Conference on Decision and Control 2004, IEEE, San Diego, CA, USA, pp. 2929-2933.

Becker, G. \& Packard, A. (1994). Robust performance of linear parametrically varying systems using parametrically-dependent linear feedback, Systems \& Control Letters 23(3): 205215.

Bokor, J. \& Balas, G. (2004). Detection filter design for LPV systems - a geometric approach, Automatica 40(3): 511-518.

Chen, B. \& Peng, H. (2001). Differential-braking-based rollover prevention for sport utility vehicles with human-in-the-loop evaluations, Vehicle System Dynamics 36(4-5): 359389.

Chen, J. \& Patton, R. (1999). Robust Model based Fault Diagnosis for Dynamic Systems, Kluwer, Boston/Dordrecht/London.

Fischer, D. \& Isermann, R. (2004). Mechatronic semi-active and active vehicle suspensions, Control Engineering Practice 12(11): 1353-1367.

Gahinet, P. (1996). Explicit controller formulas for LMI-based $\mathcal{H}_{\infty}$ synthesis, Automatica 32(7): 1007-1014.

Gertler, J. (1997). Fault detection and isolation using parity relations, Control Engineering Practice 5(5): 653-661.

Gillespie, T. (1992). Fundamentals of vehicle dynamics, Society of Automotive Engineers Inc.

Gáspár, P., Szabó, Z. \& Bokor, J. (2006). Side force coefficient estimation for the design of active brake control, Proceedings of the American Control Conference 2006, IEEE, Minneapolis, MN, USA, pp. 2927-2932.

Gáspár, P., Szabó, Z., Szederkényi, G. \& Bokor, J. (2008). Two-level controller design for an active suspension system, Proceedings of the 16th Mediterranean Conference on Control and Automation 2008, IEEE, Ajaccio-Corsica, France, pp. 232 - 237. 
Juloski, A. L., Heemels, W. P. M. H. \& Weiland, S. (2007). Observer design for a class of piecewise linear systems, International Journal of Robust and Nonlinear Control Engineering Practice 17(15): 1387-1404.

Kanev, S. \& Verhaegen, M. (2000). Controller reconfiguration for non-linear systems, Control Engineering Practice 8(11): 1223-1235.

Leith, D. \& Leithead, W. (2000). Survey of gain-scheduling analysis and design, International Journal of Control 73(11): 1001-1025.

Massoumnia, M. A. (1986). A geometric approach to the synthesis of failure detection filters, IEEE Transactions on Automatic Control 31(9): 839-846.

Packard, A. \& Balas, G. (1997). Theory and application of linear parameter varying control techniques, Proceedings of the American Control Conference 1997, Workshop I, Albuquerque, New Mexico.

Palkovics, L., Semsey, A. \& Gerum, E. (1999). Roll-over prevention system for commercial vehicles, Vehicle System Dynamics 32(4-5): 285-297.

Rough, W. \& Shamma, J. (2000). Research on gain scheduling, Automatica 36(11): 1401-1425.

Sampson, D. \& Cebon, D. (2003). Active roll control of single unit heavy road vehicles, Vehicle System Dynamics 40(4): 229-270.

Sepulchre, R., Jankovic, M. \& Kokotovic, P. (1997). Constructive Nonlinear Control, SpringerVerlag.

Sharp, R. \& Crolla, D. (1987). Road vehicle suspension system design: A review, Vehicle System Dynamics 16(3): 167-192.

Stoustrup, J. \& Niemann, H. (1998). Fault detection for nonlinear systems - a standard problem approach, Proceedings of the 37th IEEE Conference on Decision and Control 1998, Vol. 1, IEEE, Tampa, FL, USA, pp. 96-101.

Szabó, Z., Bokor, J. \& Balas, G. (2003). Inversion of LPV systems and its application to fault detection, Proceedings of the 5th IFAC Symposium on Fault Detection Supervision and Safety for Technical Processes (SAFEPROCESS) 2003, Washington, DC, USA, pp. 235240.

Szigeti, F., Vera, C., Bokor, J. \& Edelmayer, A. (2001). Inversion based fault detection and isolation, Proceedings of the 40th IEEE Conference on Decision and Control 2001, Vol. 2, IEEE, Orlando, FL, USA, pp. 1005-1010.

van der Schaft, A. J. (2000). L2-Gain and Passivity Techniques in Nonlinear Control, SpringerVerlag, Berlin.

$\mathrm{Wu}$, F. (1995). Control of linear parameter varying systems, PhD Thesis, Mechanical Engineering, University of California at Berkeley .

Wu, F. (2001). A generalized LPV system analysis and control synthesis framework, International Journal of Control 74(7): 745-759. 


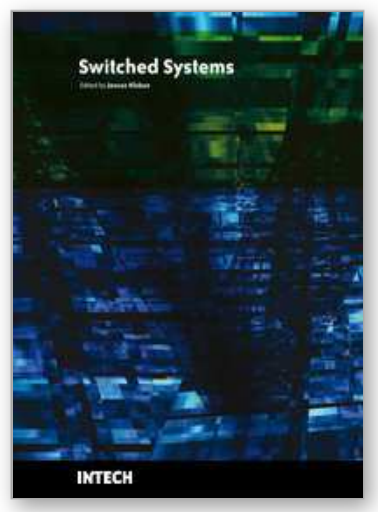

\author{
Switched Systems \\ Edited by Janusz Kleban
}

ISBN 978-953-307-018-6

Hard cover, 174 pages

Publisher InTech

Published online 01, December, 2009

Published in print edition December, 2009

This book presents selected issues related to switched systems, including practical examples of such systems. This book is intended for people interested in switched systems, especially researchers and engineers.

Graduate and undergraduate students in the area of switched systems can find this book useful to broaden their knowledge concerning control and switching systems.

\title{
How to reference
}

In order to correctly reference this scholarly work, feel free to copy and paste the following:

Peter Gaspar, Zoltan Szabo and Jozsef Bokor (2009). Active Suspension in Integrated Vehicle Control, Switched Systems, Janusz Kleban (Ed.), ISBN: 978-953-307-018-6, InTech, Available from: http://www.intechopen.com/books/switched-systems/active-suspension-in-integrated-vehicle-control

\section{INTECH}

open science | open minds

\section{InTech Europe}

University Campus STeP Ri

Slavka Krautzeka 83/A

51000 Rijeka, Croatia

Phone: +385 (51) 770447

Fax: +385 (51) 686166

www.intechopen.com

\section{InTech China}

Unit 405, Office Block, Hotel Equatorial Shanghai

No.65, Yan An Road (West), Shanghai, 200040, China

中国上海市延安西路65号上海国际贵都大饭店办公楼405单元

Phone: +86-21-62489820

Fax: $+86-21-62489821$ 
(C) 2009 The Author(s). Licensee IntechOpen. This chapter is distributed under the terms of the Creative Commons Attribution-NonCommercialShareAlike-3.0 License, which permits use, distribution and reproduction for non-commercial purposes, provided the original is properly cited and derivative works building on this content are distributed under the same license. 